\title{
On the Weak Forces on Nanoparticles
}

\author{
Diego Alducin, Miguel José-Yacamán, and Arturo Ponce
}

Department of Physics and Astronomy, University of Texas at San Antonio, San Antonio, Texas 78249, USA

"As we go down in size, there are a number interesting problems that arise”, Feynman famously said in the American Physical Society of 1959 at the California Institute of Technology. What he meant by that, was then little understood, but today as we continually research the field of nanotechnology we can quantitatively observe these "interesting problems". Weak forces, such as the Van der Waals forces, clearly affect the domain in which nanoparticles are observed. Molecular attractions, electrostatics, forces of adhesion and friction are some of the forces involved in the kinematics of nanoparticles.

In this work, we manipulated a series of capped gold nanoparticles and quantitatively measured the forces that allowed different types of motions. Combining a scanning probe tool within a transmission electron microscope we manipulated a gold nanoparticle and recorded the sliding and rolling motions of the nanoparticle across a gold surface. Determination of the contact mechanics and kinematical motion of nanoparticles is essential to improve the manipulation and understanding of materials at the nanoscale. Using in situ TEM techniques for probing the nanoparticles to measure and observe the forces involved during manipulation offered significant contributions into the way future problems can be approached.

During this experiment we introduced a Nanofactory nano-indenter holder with a silicon AFM cantilever into a transmission electron microscope. Using this holder, several nanoparticles placed on a PZT controlled gold wire was approached to the cantilever. The interaction between the cantilever, the nanoparticles and the gold wire produced different types of motions. The forces within these motions were quantified due to the deflection of the cantilever with a known spring constant $(\mathrm{k}=5.45 \mathrm{~N} / \mathrm{m})$ and due to the live visual feedback given by the recording of the motion in the TEM.

In order to study the mechanism of manipulation using the AFM tip and to measure the forces involved, initially static equilibrium conditions must be established, the free body diagram of these forces is sketched in Figure 1(b). The Hertzian model of contact mechanics with an adhesion force present between elastic bodies was first introduced by Bradley[1]. The rolling and sliding of the particles are associated to particle-substrate friction force that needs to be overcome to produce motion[2]; equivalently adhesion force plays an important role in the detachment of the contact object with the particle, either tip or substrate surface. In this way, the forces considered, based on the diagram of the Figure 1(b). The rolling motion of the particle over the surface was recorded from which a series of frames were extracted for their force analysis in Figure 2.

Our results open new insights about weak forces at individual nanoparticles in real time. The data obtained through the direct imaging of the kinematics of a nanoparticle rolling provides a more detailed and quantifiable information about these forces. The interactions between the capped nanoparticle, the tool and the surface showed how fundamental the force of adhesion is at this scale, more specifically how they change as more complex motions are introduced. This 
work will help provide more insight into how this "weak" forces are much more important at this scale.
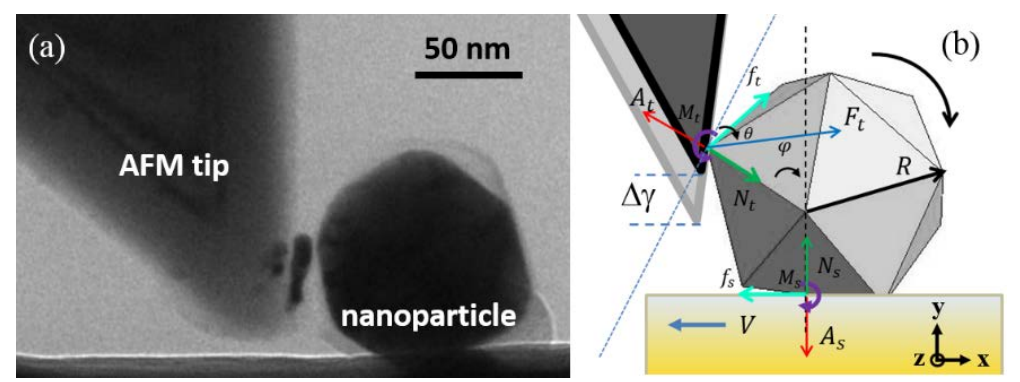

Figure 1. (a) TEM micrograph of the AFM tip approaching to a gold nanoparticle deposited in a gold wire substrate. (b) Schematic diagram of the experiment and the forces included in the manipulation of the nanoparticle.
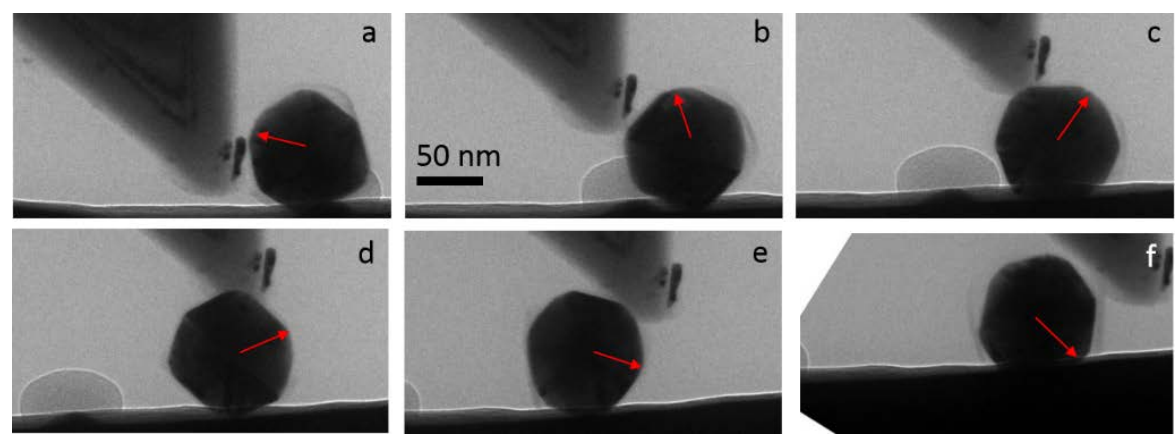

Figure 2. Rolling motion of the capped gold nanoparticle on gold surface by an AFM tip. (a) initial position, zero degrees of rolling, (b) $17.4^{\circ}$ rotation (c) $48.6^{\circ}$ rotation (d) $90.3^{\circ}$ rotation (e) $127.7^{\circ}$ rotation (f) $140.2^{\circ}$ rotation. All rotation angles are measured with respect to the initial position (a). The total displacement of the rolling is $204 \mathrm{~nm}$.

[1] Bradley RS, Phil Mag 13 (1932), p.853

[2] Saito S, et al, J Appl Phys 92 (2002), p.5140

[3] This project was supported by grants from the National Center for Research Resources (5 G12RR013646-12) and the National Institute on Minority Health and Health Disparities (G12MD007591) from the National Institutes of Health. We also thank support from NSF grants DMR-1103730 and NSF PREM Grant \# DMR 0934218. 